\title{
The bone anabolic carotenoid B-cryptoxanthin enhances transforming growth factor-B1-induced SMAD activation in MC3T3 preosteoblasts
}

\author{
MASAYOSHI YAMAGUCHI ${ }^{1}$ and M. NEALE WEITZMANN ${ }^{1,2,3}$ \\ ${ }^{1}$ Division of Endocrinology, Metabolism and Lipids; ${ }^{2}$ Winship Cancer Institute, Emory University School of Medicine, \\ Atlanta, GA 30322; ${ }^{3}$ Atlanta Veterans Affairs Medical Center, Decatur, GA 30033, USA
}

Received July 15, 2009; Accepted August 21, 2009

DOI: 10.3892/ijmm_00000278

\begin{abstract}
The xanthophyll B-cryptoxanthin is a member of the carotenoid family of plant-derived pigments endowed with anti-osteoporotic properties in vivo. B-cryptoxanthin was demonstrated to stimulate osteoblastic bone formation and simultaneously repress osteoclastic bone resorption in vitro. However, the mechanisms of action remain to be elucidated. The SMAD signal transduction pathway is established to play a critical role in osteoblast lineage commitment and differentiation. In this study we used transient transfection assays of a SMAD luciferase reporter to investigate whether ß-cryptoxanthin regulates SMAD activation in MC3T3 preosteoblastic cells. ß-cryptoxanthin did not stimulate basal SMAD activity but amplified transforming growth factor (TGF)- $\$ 1$ induced SMAD activation. Interestingly, $\beta$-cryptoxanthin did not affect bone morphogenetic protein-2 (BMP-2)-induced SMAD activation in osteoblastic cells, suggesting specificity of action on the TGF- $\beta 1$ pathway. This study suggests that the carotenoid $\beta$-cryptoxanthin may promote osteoblast differentiation and activity by amplifying TGF-B1-induced lineage commitment of osteoblast precursors.
\end{abstract}

\section{Introduction}

Bone homeostasis is maintained by a delicate balance between osteoblastic bone formation and osteoclastic bone resorption. Numerous pathological processes have the capacity to disrupt this equilibrium leading to conditions where the rate of bone resorption outpaces the rate of bone formation leading to osteoporosis, a devastating bone disease that is widely recognized as a major public health threat. Postmenopausal osteoporosis, a consequence of ovarian hormone deficiency, is

Correspondence to: Dr Masayoshi Yamaguchi, Division of Endocrinology and Metabolism and Lipids, Department of Medicine, Emory University School of Medicine, 101 Woodruff Circle, 1329 WMRB, Atlanta, GA 30322-0001, USA

E-mail: yamamasa1155@yahoo.co.jp

Key words: $\beta$-cryptoxanthin, transforming growth factor- $\beta 1, \mathrm{SMAD}$, osteoblast, bone formation, osteoporosis the archetypal osteoporotic condition in women after menopause and leads to bone destruction though complex and diverse metabolic and biochemical changes (1).

Pharmacologic and nutritional factors have long been associated with reduced postmenopausal bone loss as chemical compounds present in certain food and plants appear to modulate bone turnover. Food and plant chemical factors can help prevent bone loss but the molecular actions of these factors are poorly understood $(2,3)$.

Found predominantly in fruit and vegetables, carotenoids are a group of plant pigments whose members include $\beta$-cryptoxanthin, lutein, lycopene, astaxanthin, and $\beta$-carotene. Interestingly, $\beta$-cryptoxanthin, which is abundant in Satsuma mandarin orange (Citrus unshiu MARC.), has anabolic effects on osteoblastic bone formation (4-7) and suppressive effects on osteoclastic bone resorption in vitro $(5,8,9)$, leading to an increase in bone mass. Furthermore, oral administration of B-cryptoxanthin was shown to have anabolic effects on bone turnover in young and aged rats (10), and prevents bone loss in streptozotocin-diabetic rats (11), and ovariectomized rats (12) in vivo.

Moreover, the intake of ß-cryptoxanthin-reinforced juice for long periods of time was shown to have both stimulatory effects on osteoblastic bone formation and inhibitory effects on osteoclastic bone resorption in healthy humans and in postmenopausal women, as assessed by serum biochemical markers of bone metabolism in vivo (13). Epidemiological studies have further suggested that B-cryptoxanthin constitutes a sustainable nutritional approach to improving the bone health of human subjects (14-16).

Although B-cryptoxanthin is widely considered to be an effective nutritional factor for maintaining healthy bone, and for prevention of osteoporosis (17), the molecular mechanisms by which $\beta$-cryptoxanthin modulates bone cells are poorly understood.

ß-cryptoxanthin has been shown to have stimulatory effects on osteoblastic bone formation and mineralization in organ culture ex vivo $(4,5)$ and to increase cell number, DNA content, protein content, and alkaline phosphatase activity in osteoblastic cells $(6,7)$, suggesting that $\beta$-cryptoxanthin stimulates both preosteoblast proliferation and differentiation. $\beta$-cryptoxanthin has a stimulatory effect on the gene expression of various osteoblastic proteins (including Runx2, $\alpha 1$ (I) collagen and 
alkaline phosphatase), factors essential for osteoblastic bone formation and mineralization $(6,7)$.

How B-cryptoxanthin promotes osteoblastic differentiation is unclear, however, this factor was shown to stimulate the expression of insulin-like growth factor-I (IGF-I) and TGF- $\beta 1$ (6), factors implicated in bone growth, and in osteoblast differentiation $(18,19)$.

TGF- $\beta 1$ has potent activity on osteoblast-lineage commitment, an event that is partly mediated through SMAD transcription factors (23). We reported that NF- $\kappa \mathrm{B}$ signaling represses basal osteoblast differentiation and mineralization in MC3T3 cells and antagonizes TGFß and BMP-2 mediated MC3T3 mineralization by downregulating SMAD activation (20). Other studies found that NF-кB signaling antagonizes SMAD activation in Saos2 osteosarcoma cells by a mechanism involving induction of the inhibitory SMAD7 (21). Interestingly, we recently reported that $\beta$-cryptoxanthin has the capacity to suppress NF- $\mathrm{KB}$ activity in MC3T3 preosteoblastic cells (22) suggesting that $\beta$-cryptoxanthin leads to enhanced SMAD signaling.

In this study we examined whether B-cryptoxanthin directly stimulates SMAD activation or regulates TGF- $\beta 1$-induced SMAD activation in osteoblastic cells in vitro. Our data show that $\beta$-cryptoxanthin potentiates TGF $\beta$-induced, but not BMP-2-induced, SMAD activation in MC3T3 preosteoblastic cells, suggesting that one mechanism by which ß-cryptoxanthin stimulates bone formation is by potentiating the TGF- $\beta 1$ mediated commitment of preosteoblasts to differentiate along the osteoblastic pathway.

\section{Materials and methods}

Materials. $\alpha$-Minimal essential medium ( $\alpha$-MEM) and antibiotics (penicillin and streptomycin) were purchased from Invitrogen (Carlsbad, CA). Fetal bovine serum (FBS) was from Hyclone (Logan, UT). ß-cryptoxanthin was obtained from Extrasynthase (Lyon-Nord, France). Recombinant TGF-ß1 or BMP-2 was purchased from R\&D Systems, Inc. (Minneapolis, MN). PD 98059 was obtained from EMD Biosciences, Inc. (La Jolla, CA). Alizarin Red S, and all other chemicals were obtained from the Sigma Chemical Corporation (St. Louis, MO) unless otherwise stated.

Cell culture. The preosteoblastic cell line MC3T3-E1, clone 14 (MC3T3) was purchased from the American Type Culture Collection (Manassas, VA) and has been previously described in detail (24). Cells were cultured at $37^{\circ} \mathrm{C}$ in a humidified $5 \%$ $\mathrm{CO}_{2}$ incubator in plastic dishes containing $\alpha$-MEM supplemented with $10 \% \mathrm{FBS}$, with $2 \mathrm{mM}$ glutamine, $100 \mathrm{IU} / \mathrm{ml}$ penicillin, and $100 \mu \mathrm{g} / \mathrm{ml}$ streptomycin. Cells were passaged every 3 days using $0.2 \%$ trypsin plus $0.02 \%$ EDTA in $\mathrm{Ca}^{2+} / \mathrm{Mg}^{2+}$-free phosphate-buffered saline (PBS).

Osteoblast differentiation assays and Alizarin Red S staining. MC3T3 cells $\left(1.0 \times 10^{5}\right.$ cells per well) were cultured for $72 \mathrm{~h}$ in $\alpha$-MEM $(1.0 \mathrm{ml} /$ well) containing $10 \% \mathrm{FBS}$ in 12 -well dishes. Medium was aspirated and changed to mineralization medium [ $\alpha$-MEM supplemented with $10 \%$ FBS, L-ascorbic acid $(100 \mu \mathrm{g} / \mathrm{ml})$ and $4 \mathrm{mM} \beta$-glycerophosphate] as previously described $(7,20)$. Cells were treated with either vehicle (PBS) or $\beta$-cryptoxanthin $\left(10^{8}\right.$ or $\left.10^{-5} \mathrm{M}\right)$. Fresh medium was added every 3 days. At 21 days of culture, cells were rinsed with PBS and calcium deposition was visualized by fixing the cells in $75 \%$ ethanol for $30 \mathrm{~min}$ at $4^{\circ} \mathrm{C}$ followed by staining with Alizarin Red S (40 mM, pH 6.2) for $30 \mathrm{~min}$ at room temperature. Excess stain was removed by copious washing with distilled water.

Mineralization was quantitated following capture on a flatbed scanner (Epson Perfection 1660 Photo) by densitometry using Image J (Rasband, W.S., ImageJ, US National Institutes of Health, Bethesda, MD, USA, http://rsb.info.nih.gov/ij/, 19972009). Data are expressed in arbitrary units.

SMAD reporter constructs and luciferase assays. A SMAD reporter was generated by synthesizing a double-strand oligonucleotide bearing three tandem copies each of the consensus (5'-GTCT) SBE interspersed with its palindrome 5'-AGAC (5'-GTCTAGACGTCTAGACGTCTAGAC) (25) and cloning the oligonucleotide upstream of the luciferase gene in pGL3-BASIC (Promega, Madison, WI) to create pGL3-SMAD.

Reporter plasmids were transfected into MC3T3 preosteoblastic cells using Lipofectamine 2000 reagent (Invitrogen) as previously described (20). Briefly, MC3T3 cells ( $2 \times 10^{4}$ cells/ well) were cultured in 96-well white opaque luminometer plates (Corning Costar, Lowell, MA) for $24 \mathrm{~h}$ in $\alpha$-MEM, and then changed to $\alpha$-MEM without FBS and antibiotics and transfected with pGL3-SMAD reporter plasmid. Medium was changed after $5 \mathrm{~h}$ to $\alpha$-MEM containing antibiotics and with or without $10 \%$ FBS. Wells were treated with vehicle (PBS) or $\beta$-cryptoxanthin $\left(10^{-8}-10^{-5} \mathrm{M}\right)$, and/or TGF- $\beta 1$ (1 or $\left.10 \mathrm{ng} / \mathrm{ml}\right)$ for $24 \mathrm{~h}$. In some experiments BMP-2 $(0.5 \mu \mathrm{g} / \mathrm{ml})$ was added $16 \mathrm{~h}$ after addition of $\beta$-cryptoxanthin $\left(10^{-7}-10^{-5} \mathrm{M}\right)$, and the cells were cultured for $8 \mathrm{~h}$. Luciferase activity was measured on a microplate luminometer (Turner Designs, Sunnyvale, CA, USA) following cell lysis in Passive Lysis Buffer (Promega).

Statistical analysis. Statistical significance was determined using GraphPad InStat version 3 for Windows XP (GraphPad Software). Multiple comparisons were performed by one-way ANOVA with Tukey-Kramer post test. $\mathrm{P}<0.05$ was considered statistically significant. Data are presented as mean \pm SD of 5 replicate wells and data are representative of two or more independent experiments.

\section{Results}

$\beta$-cryptoxanthin stimulates osteoblastic mineralization. As the effect of $\beta$-cryptoxanthin on MC3T3 mineralization has not been previously reported we cultured MC3T3 cells with B-cryptoxanthin $\left(10^{-6}\right.$ or $\left.10^{-5} \mathrm{M}\right)$ for 21 days and stained for calcium deposition with Alizarin Red S. The data show that B-cryptoxanthin had a stimulatory effect on MC3T3 mineralization in vitro which was saturated at $10^{-6} \mathrm{M}$ (Fig. 1).

$\beta$-cryptoxanthin amplifies TGFß-induced SMAD activity in MC3T3 cells. B-cryptoxanthin has been shown to stimulate osteoblastic bone formation and mineralization ex vivo $(6,7)$. However, the mechanism by which this carotenoid achieves this action remains unclear. We recently reported that $\beta$-cryptoxanthin antagonizes NF- $\mathrm{KB}$ signal transduction (22), a potent 


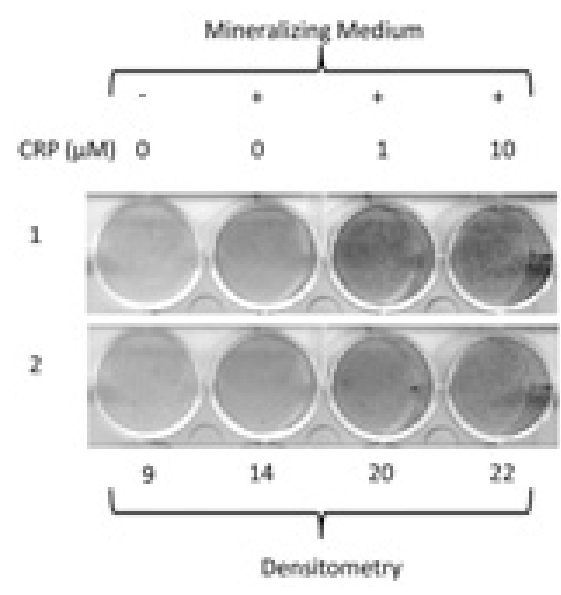

Figure 1. B-cryptoxanthin promotes osteoblastic differentiation and mineralization in preosteoblastic MC3T3 cells. MC3T3 cells were differentiated into mineralizing osteoblasts by culture in $\alpha$-MEM supplemented with $10 \%$ FBS, ascorbic acid (100 ng/ml) and $4 \mathrm{mM} \mathrm{B-glycerophosphate} \mathrm{(mineralizing}$ medium) in the presence or absence of $\beta$-cryptoxanthin (CRP) at $10^{-6}$ or $10^{-5} \mathrm{M}$. After 28 days cells were washed with PBS and stained with Alizarin Red S to visualize calcium deposition. The outcome of 2 independent experiments (labeled 1 and 2) is shown. Average densitometric quantitation is presented below each well in arbitrary units.

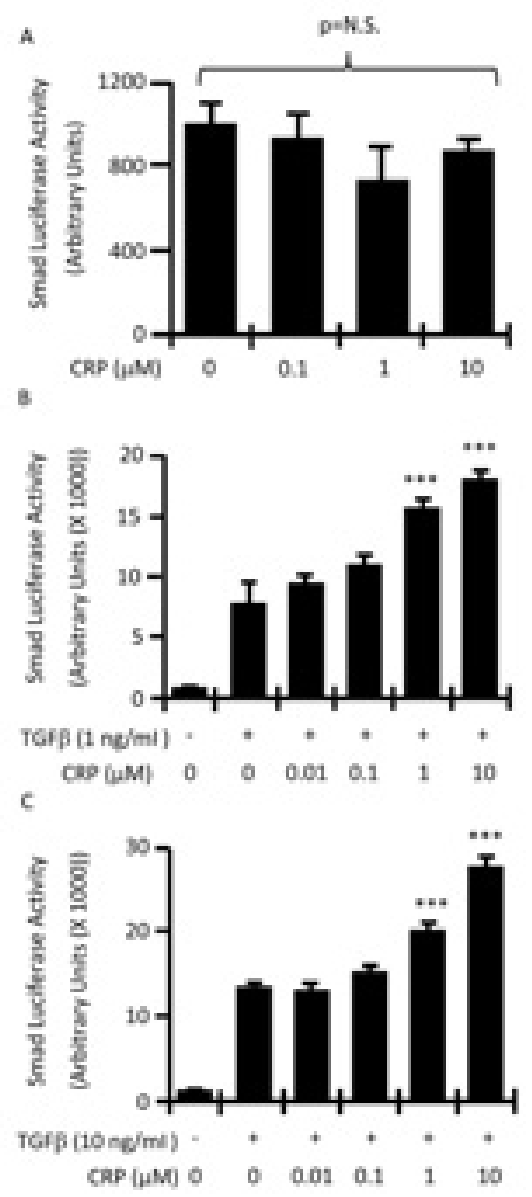

Figure 2. ß-cryptoxanthin enhances TGF- $\beta 1$-induced SMAD activation in preosteoblastic MC3T3 cells. MC3T3 cells were transfected with pGL3-SMAD, a SMAD activity reporter and cells cultured in the presence or absence of (A) B-cryptoxanthin $\left(10^{-7}-10^{-5} \mathrm{M}\right)$, (B) TGF- $\beta 1(1 \mathrm{ng} / \mathrm{ml})$ with $\beta$-cryptoxanthin $\left(10^{-8}-10^{-5} \mathrm{M}\right)$, or $(\mathrm{C})$ TGF- $\beta 1(10 \mathrm{ng} / \mathrm{ml})$ with $\beta$-cryptoxanthin $\left(10^{-8}-10^{-5} \mathrm{M}\right)$. Luciferase activity was determined after $24 \mathrm{~h}$. Data represent mean $\pm \mathrm{SD}$ of five replicate assays. ${ }^{* * *} \mathrm{P}<0.001$ relative to pGL3-SMAD + TGFß only (one-way ANOVA). N.S., no significant difference between data within brackets.

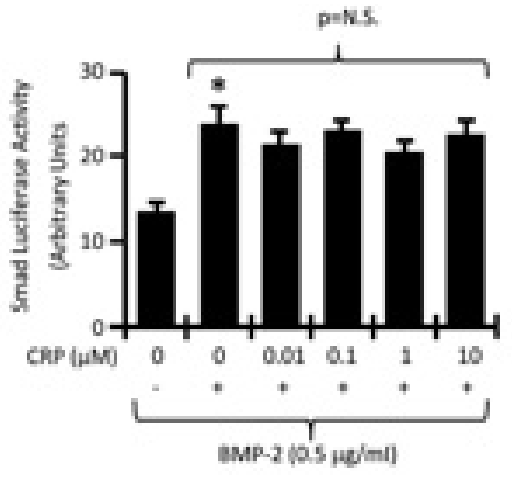

Figure 3. B-cryptoxanthin fails to enhance the BMP-2-induced SMAD activation in preosteoblastic MC3T3 cells. MC3T3 cells were transfected with pGL3-SMAD and cells cultured in the presence or absence of BMP $(0.5 \mu \mathrm{g} / \mathrm{ml})$ with or without $B$-cryptoxanthin $\left(10^{-8}-10^{-5} \mathrm{M}\right)$. Luciferase activity was determined after $24 \mathrm{~h}$ of BMP-2 addition. Data represent mean \pm SD of five assays. ${ }^{* * *} \mathrm{P}<0.001$ relative to pGL3-SMAD only (one-way ANOVA). N.S., no significant difference between data within brackets.

inhibitor of osteoblast differentiation, and a suppressor of SMAD activation. We thus investigated whether ß-cryptoxanthin is capable of promoting basal or TGFß stimulated SMAD activation, using a SMAD responsive reporter (pGL3-SMAD). While ß-cryptoxanthin $\left(10^{-7}-10^{-5} \mathrm{M}\right)$ was not seen to significantly impact basal MC3T3 SMAD activity (Fig. 2A), it significantly and dose-dependently $\left(10^{-8}-10^{-5} \mathrm{M}\right)$ enhanced TGF-B1 (1 and $10 \mathrm{ng} / \mathrm{ml})$-induced SMAD activation (Fig. 2B and C, respectively).

As BMP-2, another potent pro-osteoblastic factor, also signals via the SMAD pathway (25), we examined whether ß-cryptoxanthin could also amplify BMP-2-induced SMAD activation. Interestingly, in contrast to TGF- $\beta 1$, $ß$-cryptoxanthin $\left(10^{-8}-10^{-5} \mathrm{M}\right)$ had no potentiating effect on BMP-2-induced $(0.5 \mu \mathrm{g} / \mathrm{ml})$ SMAD activation in MC3T3 cells (Fig. 3).

As B-cryptoxanthin has been reported to promote MC3T3 proliferation and differentiation by means of a MAP kinase kinase (MEK) dependent pathway sensitive to the MEK inhibitor PD98059 (6), we examined whether PD98059 $(26,27)$ ablates the potentiating action of $\beta$-cryptoxanthin on TGFß-induced SMAD activity. PD98059 $\left(10^{-7}-10^{-5} \mathrm{M}\right)$ had no significant effect on basal SMAD activity (Fig. 4A). Furthermore, PD98059 $\left(10^{-7}\right.$ and $\left.10^{-6} \mathrm{M}\right)$ had no significant effect on the capacity of $\beta$-cryptoxanthin $\left(10^{-5} \mathrm{M}\right)$ to enhance TGF-B1 (10 ng/ml)-induced SMAD activity (Fig. 4B).

\section{Discussion}

ß-cryptoxanthin has been shown to have a stimulatory effect on osteoblastic bone formation in vitro $(6,7)$ and in vivo (10-12) by stimulating osteoblastic cell proliferation and differentiation and by regulating osteoblastic gene expression, including Runx2 a master regulator of osteoblast differentiation $(26,28)$, the matrix protein of $\alpha 1$ (I) collagen $(6,29)$, and alkaline phosphatase which participates in the mineralization process $(6,30)$. The molecular mechanism by which $B-$ cryptoxanthin promotes osteoblast differentiation is, however, poorly defined. We have previously reported that TGFß and/or BMP-2 signaling through SMADS is impeded by basal and stimulated (TNF $\alpha$ or RANKL) induced NF- $\mathrm{KB}$ 
A

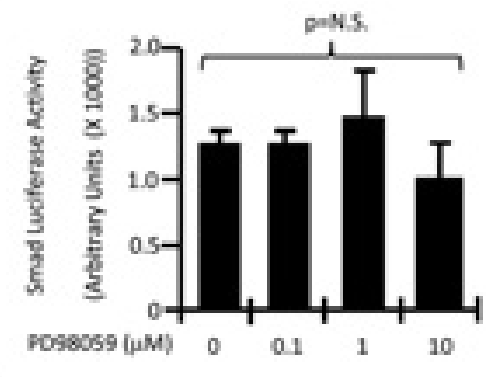

8

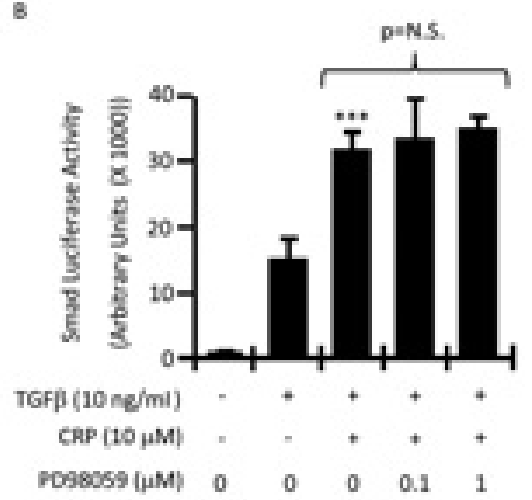

Figure 4. Inhibition of MEK fails to block ß-cryptoxanthin augmented TGF-ß1induced SMAD activation in MC3T3 preosteoblastic cells. A) MC3T3 cells were transfected with pGL3-SMAD and treated with PD98059 $\left(10^{-7}-10^{-5} \mathrm{M}\right)$ or B) with PD98059 $\left(10^{-7}-10^{-6} \mathrm{M}\right)$ with TGF-ß1 (10 ng/ml) in the presence or absence of $\beta$-cryptoxanthin $\left(10^{-5} \mathrm{M}\right)$. Luciferase activity was determined after $24 \mathrm{~h}$. Data represent mean $\pm \mathrm{SD}$ of five assays. ${ }^{* * *} \mathrm{P}<0.001$ relative to pGL3-SMAD only (one-way ANOVA). N.S., no significant difference between data within brackets.

signaling. Consequently, $\mathrm{NF}-\kappa \mathrm{B}$ antagonists stimulate osteoblast differentiation and mineralization (20). We further demonstrated that $B$-cryptoxanthin antagonizes $\mathrm{NF}-\kappa \mathrm{B}$ activity in MC3T3 preosteoblastic cells (22). These observations suggested that one mechanism by which Bcryptoxanthin may stimulate osteoblast differentiation is by promoting SMAD activation. The data presented herein are consistent with this notion. While, B-cryptoxanthin failed to directly stimulate SMAD activity, it did, however, amplify TGF-ß1-induced SMAD signaling. Interestingly, BMP-2 mediated SMAD signal transduction was not augmented by $\beta$-cryptoxanthin. The reason for this difference in response is presently unclear, however while TGF- $\beta 1$ specifically activates R-SMADS 2 and 3, BMP-2 utilizes R-SMADS 1, 5 and 8. Upon R-SMAD phosphorylation and activation these SMADS associate with a Co-SMAD (SMAD4) and the complexes translocate to the nucleus where they bind to SMAD binding elements in DNA (23). One possibility is thus that $\beta$-cryptoxanthin specifically facilitates SMAD2 or 3 expression or activation. Alternatively, $\beta$-cryptoxanthin may specifically interact with, or promote the expression of TGFß receptor components. Interestingly, $\beta$-cryptoxanthin was previously reported to stimulate the expression of TGF- $\beta 1$ mRNA in MC3T3 cells (6). Consequently, it is also possible that induction of TGF- $\beta 1$ expression by $\beta$-cryptoxanthin contributes to the amplification of SMAD signaling in our experiments. This is unlikely however, as we show that $\beta$ cryptoxanthin enhanced TGF- $\beta 1$-induced SMAD activity by about the same amount ( 2-fold) with a low dose of TGF- 31 $(1 \mathrm{ng} / \mathrm{ml})$ and with a high dose of TGF-ß1 (10 ng/ml).

Previous studies also reported that $\beta$-cryptoxanthin has the capacity to promote osteoblast differentiation through MAP kinase pathways, specifically through MEK (6). In the present study the ability of ß-cryptoxanthin to enhance TGF-ß1induced SMAD activation was not suppressed by PD98059, a specific MEK inhibitor, suggesting that the amplificatory action of $\beta$-cryptoxanthin on TGF- $\beta 1$-induced SMAD activation is mediated independently of MEK.

Taken together, our data suggest that $\beta$-cryptoxanthin stimulates osteoblast differentiation through multiple overlapping pathways, at least two of which converge on the TGFß pathway, leading to enhanced TGF- 31 production, and increased sensitivity of the TGFß-induced SMAD signal transduction pathway.

ß-cryptoxanthin is a provitamin A carotenoid that can be converted to vitamin A (retinol), a factor that was reported to independently inhibit the proliferation of osteoblastic MC3T3-E1 cells (31) by binding to RXR nuclear receptors. While it is possible that some of the observed effects of $\beta$-cryptoxanthin in our studies are mediated through its conversion to retinol, this is unlikely as previous studies have demonstrated that the stimulation of Runx 2 and $\alpha 1$ (I) collagen mRNA expression in MC3T3 cells by $\beta$-cryptoxanthin is not mimicked, or impeded, by retinol (7).

In conclusion, this study demonstrates that the bone anabolic carotenoid B-cryptoxanthin enhances TGF-B1induced SMAD activation in MC3T3 preosteoblastic cells.

\section{References}

1. Weitzmann MN and Pacifici R: Estrogen deficiency and bone loss: an inflammatory tale. J Clin Invest 116: 1186-1194, 2006.

2. Yamaguchi M: Isoflavone and bone metabolism: Its cellular mechanism and preventive role in bone loss. J Health Sci 48: 209-222, 2002.

3. Yamaguchi M: Regulatory mechanism of food factors in bone metabolism and prevention of osteoporosis. Yakugaku Zasshi 126: 1117-1137, 2006.

4. Yamaguchi M and Uchiyama S: Effect of carotenoid on calcium content and alkaline phosphatase activity in rat femoral tissues in vitro: The unique anabolic effect of B-cryptoxanthin. Biol Pharm Bull 26: 1188-1191, 2003.

5. Yamaguchi $\mathrm{M}$ and Uchiyama S: $\beta$-Cryptoxanthin stimulates bone formation and inhibits bone resorption in tissue culture in vitro. Mol Cell Biochem 258: 137-144, 2004.

6. Uchiyama S and Yamaguchi M: $\beta$-Cryptoxanthin stimulates cell proliferation and transcriptional activity in osteoblastic MC3T3-E1 cells. Int J Mol Med 15: 675-681, 2005.

7. Uchiyama S and Yamaguchi M: B-Cryptoxanthin stimulates cell differentiation and mineralization in osteoblastic MC3T3-E1 cells. J Cell Biochem 95: 1224-1234, 2005.

8. Uchiyama $\mathrm{S}$ and Yamaguchi M: Inhibitory effect of ß-cryptoxanthin on osteoclast-like cell formation in mouse marrow cultures. Biochem Pharmacol 67: 1297-1305, 2004.

9. Uchiyama S and Yamaguchi M: B-Cryptoxanthin stimulates apoptotic cell death and suppresses cell function in osteoclastic cells: Change in their related gene expression. J Cell Biochem 98: 1185-1195, 2006.

10. Uchiyama S, Sumida T and Yamaguchi M: Oral administration of B-cryptoxanthin induces anabolic effects on bone components in the femoral tissues of rats in vivo. Biol Pharm Bull 27: 232-235, 2004.

11. Uchiyama $\mathrm{S}$ and Yamaguchi $\mathrm{M}$ : Oral administration of B-cryptoxanthin prevents bone loss in streptozotocin-diabetic rats in vivo. Biol Pharm Bull 28: 1766-1769, 2005.

12. Uchiyama $\mathrm{S}$ and Yamaguchi $\mathrm{M}$ : Oral administration of ß-cryptoxanthin prevents bone loss in ovariectomized rats. Int $\mathbf{J}$ Mol Med 17: 15-20, 2006. 
13. Yamaguchi M, Igarashi A, Uchiyama S, Sugawara K, Sumida T, Morita S, Ogawa H, Nishitani $M$ and Kajimoto $Y$ : Effect of B-cryptoxanthin on circulating bone metabolic markers: Intake of juice (Citrus unshiu) supplemented with ß-cryptoxanthin has an effect in menopausal women. J Health Sci 52: 758-768, 2006.

14. Wang Y, Hodge AM, Wluka AE, English DR, Giles GG, O'Sullivan R, Forbes A and Cicuttini FM: Effect of antioxidants on knee cartilage and bone in healthy, middle-aged subjects: a cross-sectional study. Arthritis Res Ther 9: R66, 2007.

15. Sugiura M, Nakamura M, Ogawa K, Ikoma Y, Ando F and Yano M: Bone mineral density in post-menopausal female subjects is associated with serum antioxidant carotenoids. Osteoporos Int 19: 211-219-720, 2008.

16. Granado-Lorencio F, Olmedilla-Alonso B, Herrero-Barbudo C, Blanco-Navarro I and Perez-Sacristan B: Seasonal variation of serum alpha- and beta-cryptoxanthin and 25-OH-vitamin $\mathrm{D}(3)$ in women with osteoporosis. Osteoporos Int 19: 717-720, 2008.

17. Yamaguchi M: ß-Cryptoxanthin and bone metabolism: The preventive role in osteoporosis. J Health Sci 54: 356-369, 2008.

18. Centrella M, McCarthy TL and Canalis E: Receptors for insulin-like growth factor-I and -II in osteoblast-enriched cultures from fetal rat bone. Endocrinology 126: 39-44, 1990.

19. Palcy S, Bolivar I and Goltzman D: Role of activator protein 1 transcriptional activity in the regulation of gene expression by transforming growth factor $\beta 1$ and bone morphogenic protein 2 in ROS 17/2.8 osteoblast-like cells. J Bone Miner Res 15: 2352-2361, 2000.

20. Li Y, Li A, Strait K, Zhang H, Nanes MS and Weitzmann MN Endogenous TNFalpha lowers maximum peak bone mass and inhibits osteoblastic SMAD activation, through NF-kappaB. J Bone Miner Res 22: 646-655, 2007.

21. Eliseev RA, Schwarz EM, Zuscik MJ, O'Keefe RJ, Drissi H and Rosier RN: SMAD7 mediates inhibition of Saos2 osteosarcoma cell differentiation by NFkappaB. Exp Cell Res 312: 40-50, 2006.

22. Yamaguchi $\mathrm{M}$ and Weitzmann $\mathrm{MN}$ : The bone anabolic carotenoids $B$-hydroxycinnamic acid and $B$-cryptoxanthin antagonize NF-kB activation in MC3T3 preosteoblasts. Mol Med Rep 2: 641-644, 2009.
23. Janssens K, Dijke PT, Janssens S and Hul WV: Transforming growth factor- $\beta 1$ to the bone. Endocrine Rev 26: 743-774, 2005.

24. Wang D, Christensen K, Chawla K, Xiao G, Krebsbach PH and Franceschi RT: Isolation and characterization of MC3T3-E1 preosteoblast subclones with distinct in vitro and in vivo differentiation/mineralization potential. J Bone Miner Res 14: 893-903, 1999.

25. Fredderick JP, Liberati NT, Waddell DS, Shi Y and Wang XF: Transforming growth factor beta-mediated transcriptional repression of c-myc is dependent on direct binding of SMAD3 to a novel repressive SMAD binding element. Mol Cell Biol 24: 2546-2559, 2004.

26. Li B: Bone morphogenetic protein-SMAD pathway as drug targets for osteoporosis and cancer therapy. Endocr Metab Immune Disord Drug Targets 8: 208-219, 2008.

27. Lee KH and Kim JR: Reactive oxygen species the generation of urokinase plasminogen activator in human hepatoma cells via MAPK pathways after treatment with hepatocytes growth factor. Exp Mol Med 41: 180-188, 2009.

28. Komori T, Yagi H, Nomura S, Yamaguchi A, Sasaki K, Deguchi K, Shimizu Y, Bronson RT, Gao YH, Inada M, Sato M, Okamoto R, Kitamura Y, Yoshiki S and Kishimoto T: Targeted disruption of Cbfa1 results in a comple lack of bone formation owing to maturational arrest of osteoblasts. Cell 89: 755-764, 1997.

29. Lian JB, Stein GS, Canalis E, Roky PG and Boskey AL: Bone formation: Osteoblast lineage cells, growth factor, matrix protein, and the mineralization process. In: Primer on the Metabolic Bone Disease and Disorders of Mineral Metabolism. Favus MJ (ed). (4th edition). Lippincott Williams \& Wilkins Press, New York, pp14-29, 1999.

30. Majeska RJ and Wuthier RE: Studies on matrix vesicles isolated from chick epiphyseal cartilage. Association of pyrophosphatase and ATPase activities with alkaline phosphatase. Biochim Biophys Acta 391: 51-60, 1975.

31. Park CK, Ishimi Y, Ohmura M, Yamaguchi M and Ikegami S: Vitamin A and carotenoid stimulate differentiation of mouse osteoblastic cells. J Nutr Sci Vitaminol 43: 281-296, 1997. 